\title{
The Physiological and Pathological Sequelae of Paralysis of the Intercostal and Abdominal Muscles in Tetraplegic Patients
}

\author{
By J. R. Silver, M.B.B.S., M.R.C.P.Ed. and A. Moulton, M.R.C.S., L.R.C.P. \\ Liverpool Regional Paraplegic Centre, Promenade Hospital, Southport, England
}

DESPITE the formation of specialised centres to deal with all types of spinal injury there is still a high mortality from respiratory failure among patients suffering from traumatic injuries of the cervical spinal cord.

Guttmann and Frankel (I966) found an early mortality of II.8 per cent. within the first three months among 229 cervical patients admitted within 48 hours of injury over an I I-year period.

Cheshire and Coates (1966) reported an early mortality of $6 \cdot 2$ per cent. among I 29 cervical patients admitted over a 5-year period.

The most recent figures available are those of Silver and Gibbon (I968) who reported an early mortality of 29.5 per cent. among 78 cervical patients admitted over a 20-year period.

In all these series the major cause of death was respiratory failure secondary to the extensive paralysis of the respiratory muscles. Even when the acute stage of spinal shock has subsided and the dangerous first three months have passed, the tetraplegic patient is still liable to develop aspiration and hypostatic pneumonia due to the paralysis of the chief expiratory muscles, the intercostals and abdominals; respiration being entirely supported by the diaphragm and accessory muscles. Investigations of respiratory function in the past by Cameron et al. (1955) have shown that the vital capacity in tetraplegic patients is smaller than normal and affected by the posture of the patient, being greatest when the patient was tilted head down and least when tilted feet down. They attributed these postural effects to the paralysis of the abdominal muscles which allowed the intestines to bulge through the flaccid abdominal wall, the diaphragm thus assuming a lower position in the chest and having a smaller excursion.

Hemingway et al. (I958) confirmed Cameron's findings that tetraplegic patients had reduced vital capacities, being only two-thirds of the predicted normal, and found, in addition, that the maximum breathing capacity was reduced. Subsequent studies by two independent methods (Silver, I963; Bergofsky, 1964) have shown that the work of breathing in tetraplegic patients is increased. This is presumably because when the diaphragm acts as the main muscle of ventilation it is less efficient than when acting in conjunction with the abdominal and intercostal muscles. However, in the later stages, Guttmann and Silver (I965) have shown by serial electromyographic studies that the intercostal and abdominal muscles may participate in the act of breathing on a reflex basis.

There is still much to be learnt of the mechanics of breathing in the tetraplegic patient and it seemed important from both theoretical and practical viewpoints to study the movements of the chest produced in tetraplegic patients by the diaphragm and accessory muscles alone.

Clinical Material. Four normal subjects and three patients with spinal cord transections were studied. In two patients the lesion was complete at $\mathrm{C}_{5}$ 
and $\mathrm{C}_{7}$ respectively and in one patient the lesion was complete to motor power at $\mathrm{C} 8$ with some sensory sparing to $\mathrm{D}_{3}$. All the subjects and patients were male and their ages ranged between 18 and 53 years. The patients were all well rehabilitated, mobile in wheelchairs, and were studied a minimum of one year after injury.

\section{METHODS}

Equipment. Movements of the thoracic cage and the volume of air breathed were taken. The movements of the chest were measured by calipers designed by Davis and Moore (1962). The volumes of air breathed were measured by taking readings by means of a potentiometer from the balance wheel of a 6-litre waterfilled Palmer spirometer. The records were then displayed simultaneously on an Elema/Schonander Meningograph 34.

The A.P. diameters of the chest were measured at five different levels. The highest level being that between the suprasternal notch and the spine of D2. The lowest level was between the xiphisternum and the spine of D8. Intermediate measurements were taken on the manubrium and two points of the sternum with the corresponding spinal processes. Lateral measurements were taken in the mid-axillary line at the level of the fourth rib and the ninth costal cartilage.

Procedure. The subjects were initially studied seated in their wheelchairs and recordings were taken when they took successive breaths from 0.5 to 3 litres. Later the subjects lay supine or on their sides on a tilting table in X-ray department. They were studied $30^{\circ}$ head up, horizontally and $30^{\circ}$ head down. Several runs on consecutive days were carried out to accustom the subject to the procedure. A minimum of three recordings were made at each volume of air breathed.

\section{RESULTS}

Normal Subject-seated. At low levels of ventilation we confirmed Keith's (1909) findings, in that the greatest movement of the chest was in the lower part, both in the anterior and lateral diameters. There was a progressive decrease in the expansion of the chest, the higher one ascended in both diameters, the upper part of the chest being relatively immobile when small breaths were taken (fig. I).

However, when larger breaths were taken the apices of the chest expanded but still did not move as much as the lower part. In no subject was a negative movement observed. These findings are in accordance with the theory of the bucket handle movement of the ribs and the reserve mechanism of respiration was the expansion of the upper part of the chest by the accessory muscles.

Patients with Cervical Injuries. The pattern of movement in the cervical patients was in complete contrast to the normal subjects. Instead of the lower part of the chest showing the greatest positive movement and expanding at all levels of ventilation, it was the upper part of the chest which moved the most. The lower part of the chest moved very little and there was a well marked sucking in of the lower ribs when maximum breaths were taken (fig. 2). This sucking in was localised to one part of the chest wall, and whilst it was constant for a given patient, it was not found in the same position in all three patients, e.g. one patient showed slight expansion of the A.P. diameter with constriction of the latera 
diameter and another showed expansion of the lateral with constriction of the A.P. diameter. Whilst the third patient showed sucking in at a slightly higher lateral level.

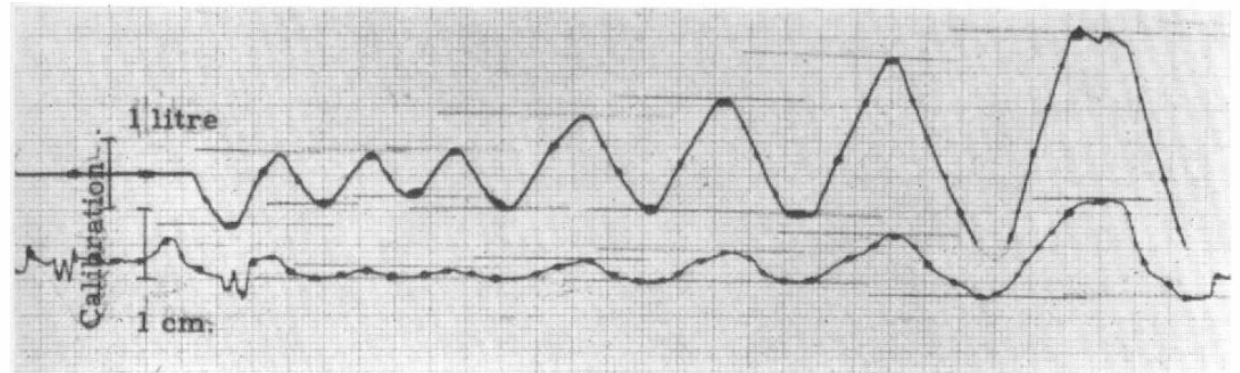

FIG. I

Tracing of Normal Subject. Upper tracing represents ventilation, inspiration upwards. Lower tracing represents expansion in the upper lateral chest. It can be seen that there is little expansion of the chest with breaths up to 5 litres but there are large positive excursions with maximum breaths.

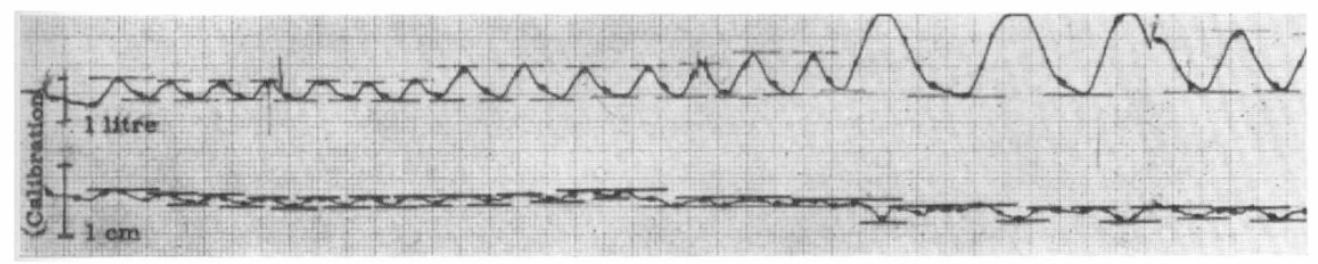

FIG. 2

Tracing of a Cervical Patient. Mid Lateral Chest. Upper tracing-spirometer. Lower tracing-caliper. With small breaths there was slight positive movement of the chest but with breaths of I litre or more the chest was sucked in. In contrast with the normal

(fig. I) where the movements are greater and positive at all levels of ventilation.

Unfortunately our equipment could measure only one diameter at a given time and so we were unable to evaluate the circumferential measurement and integrate our findings.

\section{Studies in Different Positions Relative to Gravity on a Tilting Table.}

Normal. Only one normal subject was tilted but it was found that there was no significant change in the movement of the chest in the different positions for comparable breaths (fig. 3 ).

Cervical. The cervical patients, in contrast, showed marked postural variations in the movement of the chest. When the feet were up two of the subjects showed expansion of the lower lateral chest with progressively deeper breaths, whereas when the feet were down or the patient was horizontal the findings were similar to those seen when the patient was seated, i.e., the chest was sucked in.

The upper lateral part of the chest showed minimal postural effects and the movements were mainly negative in all positions of the patient (fig. 4). The A.P. 
diameter of the chest showed a reversed postural effect to the lateral. The movements were negative with small breaths or when the patient was horizontal or feet up but became positive when the feet were down and large breaths were taken.

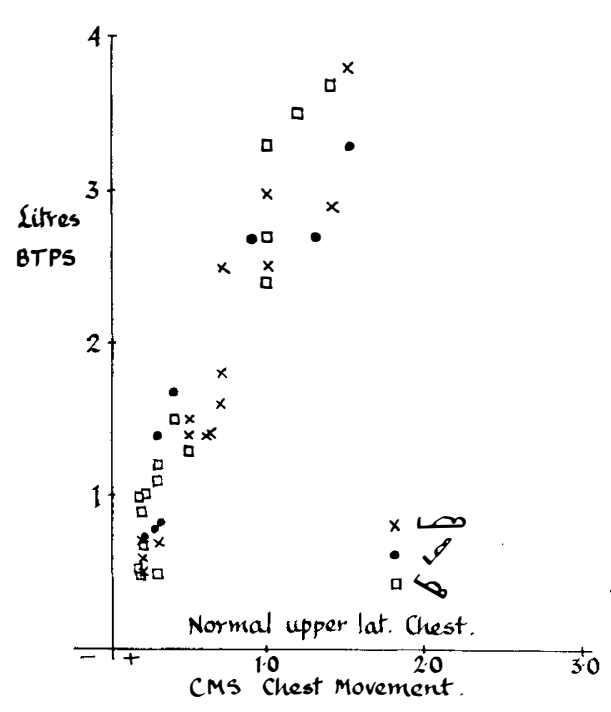

FIG. 3

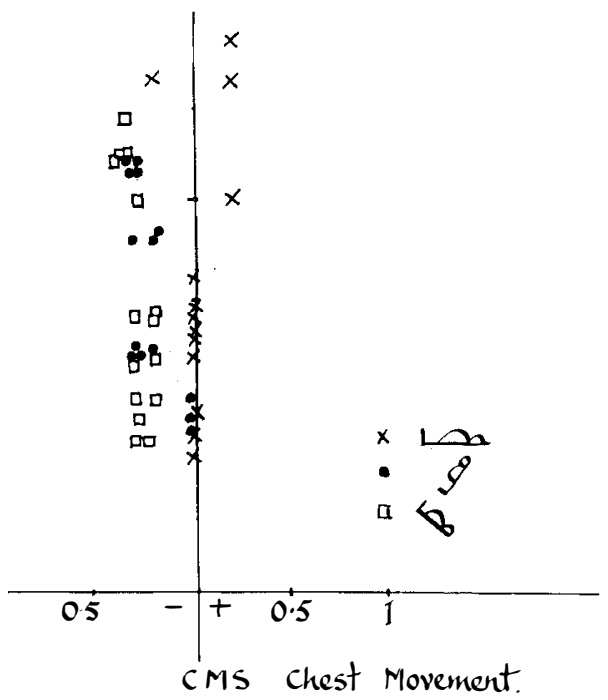

FIG. 4

FIG. 3-Recordings of the Lower Lateral Chest in a Normal Subject. Results of tilting $30^{\circ}$ feet up, horizontally and $30^{\circ}$ feet down expressed graphically. The results are positive at all levels of ventilation and do not change with the position of the patient.

FIG. 4-Recordings of the Upper Lateral Chest in a Tetraplegic Patient. Results of tilting $30^{\circ}$ feet up, horizontally and $30^{\circ}$ feet down expressed graphically. The movements remain negative, except with large breaths in the horizontal position.

\section{DISCUSSION}

These findings confirm that the chest is far from being a rigid structure which serves as a fixed attachment for the muscles. When stressed it is either expanded or constricted by the actions of the various muscles attached to it whilst at the same time depending upon these muscles for support and strength (Davis and Troup, I966).

The changes observed in the cord lesions were partly due to the loss of support of various muscles, which allowed the weight of the abdominal viscera to alter the shape of the chest, but also due to the unopposed action of the intact muscles; chiefly the diaphragm and the upper chest muscles.

In the normal subjects the greatest expansion of the chest for a given breath was in the A.P. diameter at the xiphisternum and at the lower part laterally. Tilting the only normal subject studied in this way failed to demonstrate any significant alteration in the different expansions of the chest at any given breath in the different positions, thus the normally innervated chest can compensate for the effects of gravity in the different positions studied. Just as the normal person does not develop postural hypotension under identical conditions. We were 


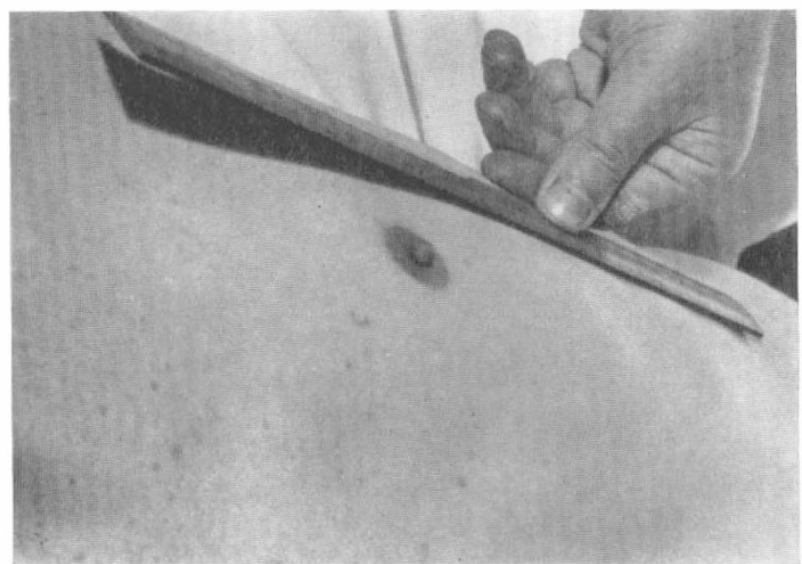

FIG. 5

Photograph of a Tetraplegic Patient. This shows the sucking in of the upper lateral part of the chest during full inspiration.

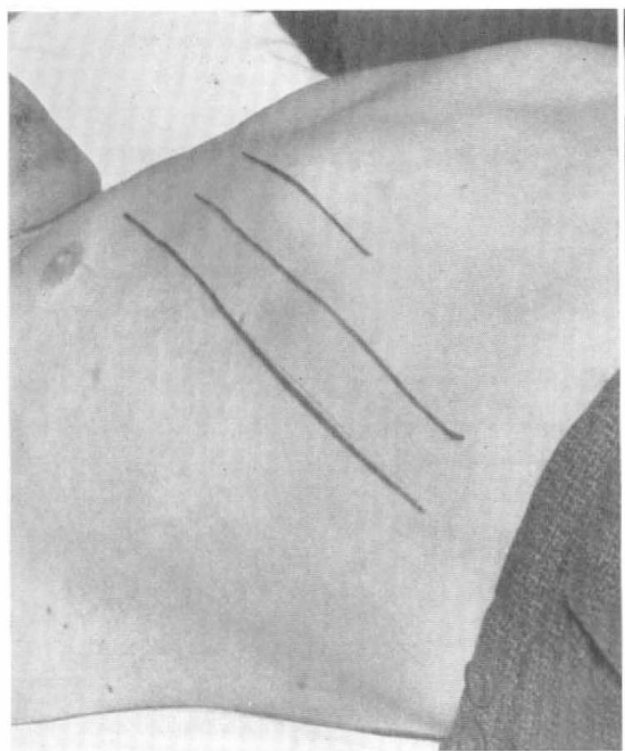

FIG. 6

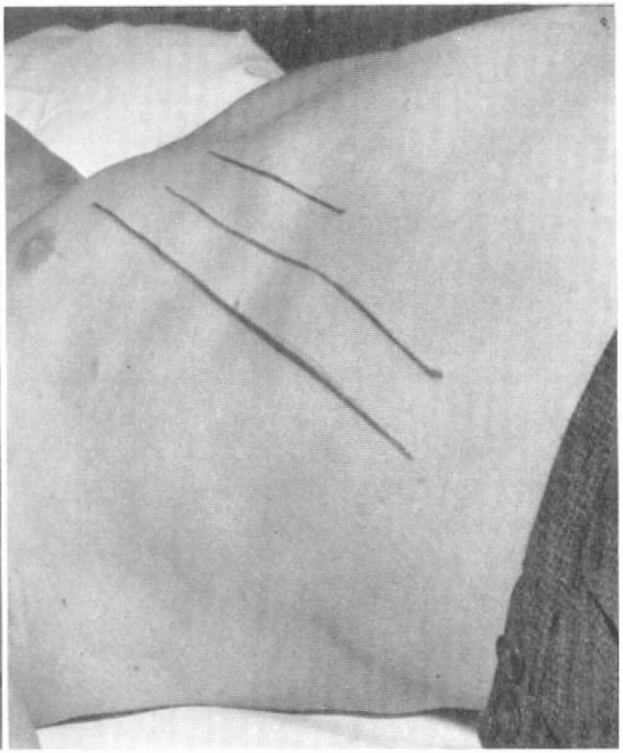

FIG. 7

Photographs of a Tetraplegic Patient During Early and Late Inspiration. The sucking in of the chest is emphasised by the distortion of the straight parallel lines on the chest. This is more marked in late inspiration. 
not in a position to determine whether there was any overall change in the chest diameters, as we were only measuring relative movements in one diameter at a time and not absolute sizes of the chest.

The cervical patients, in addition to their total loss of intercostal muscles, had also lost the function in the upper body cuirass of muscles. Inspiration pushed the abdominal contents further down. The abdominal musculature being paralysed did not oppose this and thus the abdomen was seen to bulge. The lower ribs and xiphisternum were sucked in. The position of the caliper was critical in these cases. The deformity of the ribs was variable but chiefly in the anterior portion of the lower chest, but unless care was taken in the placement of the calipers the bulging of the abdominal contents with deep inspiration could produce a positive deflection instead of a negative one. This is one of the reasons why the older methods, such as the tape measure and other circumferential devices have been unsatisf actory. It can be readily appreciated by eye and by photograph (figs. 5, 6 and 7), but is difficult to measure. It was confirmed by screening; when the lower ribs were seen to suck in on inspiration.

The sucking in of the ribs due to the low position of the diaphragm and its unopposed action in patients with paralysis of the abdominal wall was first elucidated by Duchenne (1867) both by clinical observation on patients and experiments on eviscerated animals. Keith (1909) quotes Wenckebach who described dysfunction of the diaphragm of a similar nature in visceroptosis and Campbell (1958) discussed its significance in patients with gross emphysema when he considered the low position of the diaphragm and the indrawing of the chest with inspiration contributed to the respiratory disability since it rendered the diaphragm less efficient. Boshes (1962) described this phenomena of paradoxical movement in acute cervical cord injuries but stated that it disappeared when the muscles of the rib cage and trunk passed out of the stage of spinal shock and became spastic. This sign was also described by Sandor (1966) who observed this chest movement in two patients with fresh cervical fractures in the Casualty Department and he thought it to be pathognomonic of cervical cord injuries.

Confirmation of these views is furnished by the postural effects seen in the tetraplegics. When the feet were elevated the diaphragm was pushed up by the weight of the abdominal contents, the diaphragm had a fulcrum to act about and the weight of the abdominal contents replaced the paralysed abdominal muscles. Thus the lower lateral part of the chest expanded on inspiration. In this position the complementary finding was that the lower one-third of the sternum and the xiphisternum were sucked in. When the patient was tilted feet down or in the horizontal position the abdominal contents assumed a lower position due to the paralysis of the abdominal muscles, the ribs were then sucked in at all levels of ventilation but the sternum and the xiphisternum were pushed out due to the direct pressure of the abdominal contents. The upper lateral chest being far removed from the direct action of the abdominal contents showed no postural effects and remained negative in nearly all patients, in all positions.

In contrast to the lower part of the chest which is affected by the position of the abdominal contents, the apices were affected by two main factors: the actions of the intact accessory muscles and the position of the arms, which fixed the pectoral girdle. With small breaths the negative effect of the diaphragm could be detected. However, with larger breaths, when the accessory muscles were mobilised, the upper part of the chest expanded. The upper part of the chest is 
far removed from the region of the diaphragm, and it would seem that the diaphragm could not exert a direct pull upon this part of the chest. The negative effect on the upper part of the chest is persumably due to the diaphragm acting to produce a negative intra-thoracic pressure, which would then draw in the ribs (figs. 8 and 9).

When the patient was tilted head down the movements at the apices were smaller, this could have been due to the head being flexed due to the uncomfortable position, or the clavicle could have assumed a higher position, thus rendering the sternomastoid less efficient. When the patient was tilted feet down the sternomastoids acted more efficiently and expanded the chest. Again it was found that when the arms were fixed by locking them behind the chair there was a greater expansion of the chest than when the arms were allowed to rest floppily by the side. This is a well known phenomena, due to the fixation of the insertion of latissimus dorsi and pectoralis major muscles-the origin in the upper chest thus moving.

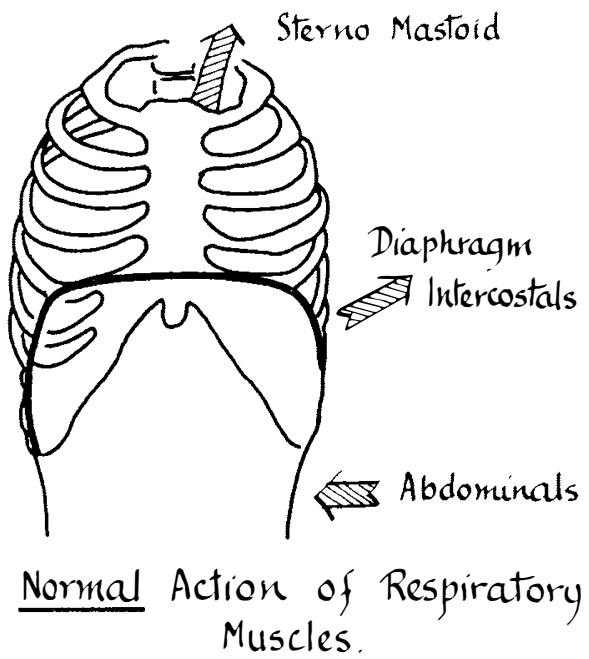

FIG. 8

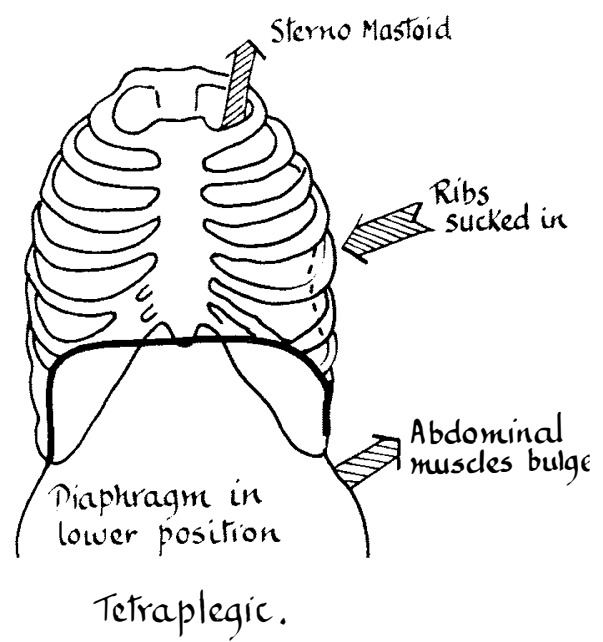

FIG. 9

FIG. 8-Diagram of the Normal Action of the Respiratory Muscles on the Rib Cage and Abdominal Wall. The abdominal muscles contract at full inspiration opposing the descent of the abdominal contents and thus provide a fulcrum against which the diaphragm can act.

FIG. 9-Diagram of the Supposed Forces Acting in a Tetraplegic Patient upon the Rib Cage and Abdominal Muscles. The flaccid abdominal muscles allow the abdominal viscera to sink down and thus the diaphragm has no fulcrum to act against. The low position of the diaphragm pulls the ribs inwards.

The functional effects of the paralysis of the intercostal and abdominal muscles is reflected in the reduced vital capacity, the inability to cough and the high mortality in the acute stages of spinal shock immediately after a traumatic injury to the cervical cord. At this time the lesion may ascend a segment or two due to local oedema and there may be other associated injuries, particularly a head injury, that depress respiration. However, even when the acute stage of the spinal shock has passed there is still a liability to develop aspiration or hypostatic pneumonia, particularly after an upper respiratory tract infection. Tribe (1963) found in I50 post mortems at the National Spinal Injuries Centre, Stoke 
Mandeville Hospital, that eight patients, all cervical injuries, had died of respiratory failure within two months of injury and a further four had died after this period, of respiratory failure.

Silver and Gibbon (I968) in an analysis of the prognosis of the I4I cases seen at Liverpool between 1947 and 1967 found that 21 patients died of respiratory failure and one of pneumonia within three months of injury and that a further five died of pneumonia subsequently. The importance of the paralysis of the respiratory muscles is reflected in the observation that the majority of the deaths-I7-occurred in complete lesions although there were only 45 complete patients admitted, compared with 96 incomplete lesions. The dangers of a simple cold or upper respiratory tract infection cannot be over-stressed and all tetraplegic patients commence postural drainage, inhalations and a broad spectrum antibiotic when they develop a cold, to avoid these dangers.

A point that is perhaps not appreciated is the much higher mortality among cervical patients should they develop a pulmonary embolus. Walshe and Tribe (1965) and Watson (1968) have shown that there is no higher incidence of venous thrombosis in the legs among the cervical patients compared with any other group of paraplegic patients; both publications showed that the highest incidence of deep vein thrombosis was in the thoracic lesions. But when a complete cervical patient develops a pulmonary embolus it is almost invariably fatal. Watson (I968) showed that all three pulmonary emboli in the complete cervical injuries were fatal, compared with the five emboli in the incomplete cervical lesions, none of which was fatal. In the recent study (Silver and Gibbon, 1968) two of the three deaths from pulmonary emboli were in complete lesions.

This is probably because while the incidence of deep vein thrombosis and pulmonary emboli is similar in all groups of patients at all levels, whether complete or incomplete, there is no reason to suppose that the size of the embolus differs. But the patients with complete cervical injuries have the smallest vital capacity. In the acute stage of spinal shock tetraplegic patients may have a vital capacity as small as $300 \mathrm{ml}$. Consequently a pulmonary embolus that falls on such a small amount of functioning lung tissue and reduces it by 50 per cent. will be immediately fatal, whereas in the case of a low lesion a similar pulmonary embolus that falls on a functioning lung volume of say $2 \frac{1}{2}$ litres will still leave the patient with a viable reserve of function.

Unfortunately it is not easy to compensate for the paralysis of the abdominal muscles by the use of tight binders, since the reciprocal relationship between the abdominal muscles and the lower chest entails that an effective binder will also compress the lower chest. The patient's ability to cough is thus impaired. The patients can compensate by leaning forward to compress their abdominal muscles by their chest.

There are still many problems to be solved regarding the movements of the chest, the pressures generated and the participation of the paralysed muscles in the act of respiration. These are being pursued by correlating the abnormal movements described above with the electromyography of the intercostal and abdominal muscles.

\section{A.CKNOWLEDGEMENTS}

One of us, J.R.S., wishes to thank the Action for the Crippled Child for a grant with which this work was carried out. We would like to thank Professor P. R. Davis of the 
University of Surrey for the loan of the calipers, Dr. K. F. Morle, Consultant Radiologist, for screening the patients and Dr. E. G. N. Campbell for his initial advice. We would also like to thank Mr. L. Henry of Sierex Ltd., for building the potentiometer circuit for the respiratory recordings and for his assistance during the tilting experiments.

This work was carried out at the Liverpool Regional Paraplegic Centre, Promenade Hospital, Southport which is part of the Southport and District Hospital Management Committee.

\section{REFERENCES}

Bergofsky, (1964). Ann. of Int. Med. 6I, 3, p. 435.

Boshes, B. (1962). Trauma to the Spinal Cord. Clin. Neurol. (Ed. A. B. Baker). New York: Harper Bros.

Cameron, G. S., Scott, J. W., Jousse, A. T. \& Botterill, E. H. (I955). Ann. Surg. I4I, $45 \mathrm{I}$.

CAMPBELl, E. J. M. (1958). The Respiratory Muscles. London: Lloyd Luke.

Cheshire, D. J. E. \& Coates, D. A. (I966). Int. F. Paraplegia, 4, I.

Davis, P. R. \& Moore, R. E. (I962). Proc. Phys. Soc. F. Phys. I62, 8.

Davis, P. R. \& Troup, J. O. G. (I966). F. Anatomy, I00, (2) p. 397.

Duchenne, G. B. A. (1867). Physiology of Motion. (Ed. E. B. Kaplan) Lippincott: Phil. I949.

Guttmann, L. \& Frankel, H. (1966). Int. F. Paraplegia, 4, 63.

Guttmann, L. \& Silver, J. R. (1965). Int. F. Paraplegia, 2, I.

Hemingway, A., Bors, E. \& Hobby, R. P. (I958). F. Clin. Invest. 37, 775.

KeIth, A. (1909). In Further Advances in Phys. (Ed. Leonard Hill). London: Edward Arnold.

SANDOR, F. (1966). B.M.f. 1, p. 465.

Silver, J. R. (1963). Int. F. Paraplegia, I, 204.

Silver, J. R. \& Gibbon, N. O. K. (I968). B.M.f. 4, 47-8.

Tribe, C. R. (1963). Int. F. Paraplegia, $\mathbf{1}, 19$.

Walsh, J. J. \& TRIBe, C. R. (I965). Int. F. Paraplegia, 3, 209.

Watson, N. (1968). Int. F. Paraplegia, 6, I I 3.

\section{Discussion}

Dr. H. L. Frankel (Great Britain). I'd like first to congratulate John Silver on this fine accurate bit of work done, obviously with a very simple and presumably cheap apparatus ... (Dr. Silver interjects: Three thousand pounds...) Well, simple apparatus.

I should like to ask him a technical point. Are these calipers fixed to the skin? How are they located, to prevent errors so that then the accessory muscles of respiration pull the chest upwards you are not just presenting a new and wider bit of the chest to your measurement? Can you just clarify for me?

Dr. J. Silver (Great Britain). The calipers were not designed by me; they were designed by Professor Davies who was at the Royal Free and is now at the University of Surrey. He leant me the calipers. You screw them up with a couple of bolts, with rubber phalanges on the actual calipers; then you put the caliper on to the chest with another part on to the spine at the back. You then tighten the nuts so that the thing is in firm contact with the bony prominences the whole time. Then when the patient takes a deep breath there is a distortion of the calipers but they don't shift on the skin. The assistant holds the hinge of the calipers in his hand the whole time.

Professor Davies designed these calipers to study coal-miners and dockers at work, so that they could be recorded continuously while working. There isn't a shift of the actual caliper on the skin. The whole chest may shift up and down. 
Dr. KERR (Great Britain). I should like to make a suggestion for a further experiment that Dr. Silver might carry out in the normal subject—and that is: the trained professional singer. I seem to remember, from the dim and distant past, that the diaphragm holds the abdominal contents with the abdominal muscles and allows the intercostals to expand the chest in the normal subject. In a trained professional singer the diaphragm is used for singing and you have got diaphragmatic breathing in which the singer allows the abdomen to expand as the diaphragm goes down, and you have a graduated upward and downward movement of the diaphragm with very little intercostal movement. This, I think, would be worth studying in addition to his other subjects.

Mr. T. McSweEnEy (Great Britain). I'd like to congratulate John Silver on not only a scientific but a most philosophical paper. It's a far cry from the opening paper yesterday morning-but I'm quite certain that the physiology which you've brought to our attention has something to do with the development of this dreadful scoliosis we see in tetraplegic children.

We know from experimental studies that rib pressures and, in partiuclar, the pressure at the costal-vertebral junction is an aetiological factor in scoliosis-so-called idiopathic scoliosis-and must have a bearing on our own problems.

Another small point-people are suggesting to you further experimental work, but I wondered whether you'll have an opportunity to observe such a chest in a tetraplegic patient under positive pressure of ventilation. Because I'm sure, again, that the mechanism you have described is not too dissimilar from traumatic paradoxical respiration, where positive pressure alters the whole physiology.

Dr. Talbot (U.S.A.). Dr. Silver's very elegant piece of work, which I hope he will continue, brings to mind one relevant clinical application. He referred to the contracture of the intercostal muscles and the suggestion of the X-ray films where you see the ribs brought together under a sort of pyraform deformity of the thoracic cage. I must remind you that this deformity, like any other contracture deformity, can be avoided, and that in all large centres, of course, where we are aware of this, there is at least one physical therapist whose sole duty is to take care of avoiding this deformity of the thoracic cage by appropriate exercise, active and passive. It is important to remember this in areas and hospitals where smaller groups of patients are taken care of and where it is perhaps not possible to assign one therapist exclusively to this duty. But one of them should have this at least as part of her work because this, like any other contracture deformity, can be avoided to the great benefit of the patient, especially in terms of his pulmonary compliance later on.

Sir Ludwig GutTmanN (Great Btitain). From the beginning in our work on tetraplegics it was found that although the vital capacity of the lung in the acute stages of tetraplegia was, say, 500 or even sometimes only 100, in later stages it increased to 2 litres and even more. That puzzled me in I 944 and I asked myself: 'Why is it?' Of course, I found very quickly that it was due to the action of the auxiliary respiratory muscles which the Almighty or nature has given us. The muscles which are of greatest importance are the sterno-cleido-mastoids, the platysma and trapezius. Their combined function lift the upper part of the thoracic cage upwards and forwards thus increasing the antero-posterior diameter of the lung. Therefore these muscle groups have to be exercised by the physiotherapist from the beginning of the tetraplegia.

If you do this, you will find that even in a few weeks these muscles develop a hypertrophy and their power to improve the ventilation greatly increases. That is a very important point in the restoration of the capacity of the lung and I am glad that John Silver has now confirmed that by his excellent piece of research which I hope he will continue. He also has gone back into the history of the research on muscle movement by quoting the work of Duchenne de Boulogne. I would suggest that people who are 
concerned with the physiotherapy in tetraplegia and paraplegia should really read this book. There is an English translation of it. You will find how this great man, in the middle of the last century, by electrical stimulation of individual muscles, made a profound contribution to our knowledge of muscle function.

Mr. N. GibBon (Great Britain). I would just like to say that in these days when it is sometimes said at home that it is impossible to do research work because one hasn't got the accommodation, one hasn't got the staff, one hasn't got the equipment and so on, that people ought to know just how this work was done. I go into Dr. Silver's office every week and I have to step over lumps of expensive equipment on the floor. All this is done in his little office about Io ft. by $6 \mathrm{ft}$. 6 in. There are no rooms available in the hospital for research work. He has had it all installed in there-the patients are brought in, and there is literally no room to move. It is not the accommodation, it is the chap who is doing the work-and there's a lesson for that everywhere in the world: when there is perhaps lack of money and lack of accommodation it is no excuse really for not getting down to the basic work.

One other small point about this. I believe that a hospital casualty officer wrote up to the journals the other day and pointed out that this simple physical sign-in-drawing of the chest-could be used to distinguish in an unconscious patient with a head injury that he also had an injury in the cervical spine. He had made this simple clinical observation-it may not have been original-but I think it was an important point.

Dr. J. R. SIlver (Great Britain). I would just like to thank people for their kind remarks and deal with one or two of the points.

The question of the singers and the movement of the diaphragm is, I think, a very complex one that has been studied. There is a book on the Physiotherapy of Exercise by Licht where it is discussed, whether trained singers can, in fact, control the diaphragm and their intercostal muscles. What the exact results were I am not sure, but it wasn't quite, I think, what was thought before.

Dr. Talbot raised a very important point-the question of the physiotherapy as a regular routine. All our tetraplegics on admission actually, as at Stoke Mandeville and the other Spinal Units, get routine physiotherapy, breathing exercises-and later as soon as they get a cold the regime recommences.

Mr. McSweeney raised the point about the contribution of this to spinal deformities. In fact I entitled it: the Abnormal Forces ... and I thought it would fit in with yesterday's papers, because I think one needs to do the electromyography of the longer recto-spinal muscles to study these points.

I am sorry that I didn't mention Dr. Sandor who is the casualty officer who made this observation to which Mr. Gibbon drew attention. 